\title{
Alternativa de abordagem endovascular para hematoma em expansão pós-punção arterial: relato de dois casos
}

\section{Alternative endovascular approach for expanding hematoma after arterial puncture: report of two cases}

Marco Aurélio Barroso Aguiar Filho․ Frederico Augusto de Carvalho Linhares Filho ${ }^{1}$. João Edison de Andrade Filho ${ }^{1}$. Matheus Duarte Pimentel ${ }^{1}$. Francisca Jovita de Oliveira Veras Albuquerque ${ }^{1}$. Carmelo Silveira Carneiro Leão Filho ${ }^{1}$.

1 Universidade Federal do Ceará (UFC), Fortaleza, Ceará, Brasil.

\section{RESUMO}

O crescente número de procedimentos endovasculares realizados mundialmente fez com que seja mais notável a incidência de complicações iatrogênicas decorrentes de punções arteriais, com taxas desses eventos variando de 0,5 a $11 \%$. Abordagens convencionais com compressão local, uso de dispositivos de fechamento de punção, são utilizadas com frequência, mas alguns casos mais complexos requerem abordagem cirúrgica. Técnicas endovasculares mostram-se como alternativas cada vez mais descritas para reparo cirúrgico destas complicações por sua eficácia, segurança e menor morbidade. Nesse relato ilustramos casos de dois pacientes portadores de hematomas expansivos pós-punção arterial nos quais foi empregada, com sucesso, técnica de insuflação de cateter-balão para hemostasia endovascular dos pontos de hemorragia arterial. Esta abordagem foi satisfatória, com resolução da complicação vascular e evitando maior morbidade.

Palavras-chave: Procedimentos endovasculares. Cateterismo periférico. Doença arterial periférica. Hematoma.

\section{ABSTRACT}

The increasing number of endovascular procedures performed worldwide made the incidence of iatrogenic complications from arterial punctures more relevant, with rate of these events ranging from 0.5 to $11 \%$. Conventional approaches involving local compression, use of puncture closure devices, are frequently used, but in cases with higher complexity, a surgical approach is usually required. Endovascular techniques are increasingly described as alternatives for surgical repair of these complications due to their efficacy, safety and lower morbidity. In this report, we illustrate the cases of two patients with post-puncture expansive hematomas in which a technique of balloon catheter insufflation for endovascular hemostasis of the arterial hemorrhage points was successfully used. This approach was satisfactory, with resolution of the vascular complication, and avoiding greater morbidity.

Keywords: Endovascular procedures. Peripheral catheterization. Peripheral artery disease. Hematoma.

Autor correspondente: Marco Aurélio Barroso Aguiar Filho, Rua Professor Francisco Gonçalves, 1271, Aldeota, Fortaleza, Ceará. CEP: $60192-170$. Telefone: +55 85 99999-2119. E-mail: drmarcovascular@gmail.com

Conflito de interesses: Não há qualquer conflito de interesses por parte de qualquer um dos autores.

Recebido em: 23 Dez 2018; Revisado em: 14 Mar 2019; Aceito em: 08 Abr 2019. 


\section{INTRODUÇÃO}

O número de procedimentos cirúrgicos endovasculares apresenta crescente aumento nos últimos anos. Associado a isto, tornou-se mais frequente a necessidade de punções arteriais femorais para procedimentos diagnósticos e terapêuticos da medicina intervencionista, com os índices de complicações iatrogênicas tornando-se mais relevantes, uma vez que estas técnicas de punção apresentam elevado nível de complexidade, com longa curva de aprendizado e seguindo em adaptação. ${ }^{1-4}$

Em grandes centros, a incidência de complicações ainda varia de 0,5 a $11 \%$, a depender de fatores procedurais, e de fatores de risco apresentados pelos pacientes. ${ }^{1,2,3,5}$ Estes eventos decorrentes dos acessos arteriais incluem complicações vasculares como: hemorragias, formação de hematomas, de pseudoaneurismas, de fístulas arteriovenosas, bem como ocorrência de trombose, embolização distal, e dissecção arterial. ${ }^{1,3,5}$ Complicações não vasculares como dano a nervos, infecção local e formação de linfocele também são relatadas. ${ }^{1,3}$

Quando ocorrem complicações vasculares como as descritas anteriormente, cerca de $21 \%$ dos pacientes necessitam de correção cirúrgica para evitar complicações maiores. ${ }^{1,5}$ Dessa forma, o desenvolvimento de novas abordagens menos invasivas, sobretudo por via endovascular, vem sendo aspecto enfocado por diversas instituições visando resolução destes eventos adversos com menor morbidade e mortalidade..$^{3-7}$

O objetivo deste relato é ilustrar dois casos de pacientes portadores de hematoma expansivo pós-punção arterial nos quais abordagens endovasculares alternativas foram empregadas para correção do quadro.

\section{RELATOS DE CASO}

\section{Caso 1}

Paciente de 83 anos, sexo feminino, hipertensa, diabética, extabagista, apresentava doença arterial obstrutiva periférica (DAOP) dos eixos aorto-ilíaco e fêmoro-poplíteo com lesão trófica do membro inferior direito. Submetida a tratamento endovascular de DAOP com proposta de punção anterógrada da artéria femoral comum direita. Evoluiu no intraoperatório com hematoma em expansão na região inguinal e retroperitoneal, além de aumento sensível de frequência cardíaca e queda de pressão arterial sem configurar um choque hipovolêmico, respondendo satisfatoriamente à reposição volêmica. Realizado arteriografia de controle que evidenciou extravasamento de contraste em artéria ilíaca externa por orifício causado por tentativas de punções no procedimento em questão. Por ser um local de compressão impossibilitada, pelo fato do serviço não dispor de stent revestido (realidade de muitos serviços de hospital público), foi optado por realizar punção de artéria braquial esquerda (punção de femoral comum contra-lateral seguida de "cross-over" impossibilitada porque a paciente realizou um "kissing-stent" previamente) com aposicionamento de introdutor $5 \mathrm{~F}$ sob a técnica de Seldinger, cateterização de artéria ilíaca externa direita com guia hidrofílico 0,035 ”x260 $\mathrm{cm}$ e cateter Berenstein 5F. Realizado arteriografia pelo cateter para confirmação do local do sangramento. Posicionado cateter-balão semi-complacente $6 \times 60 \mathrm{~mm}$, sendo insuflado por 3 minutos para realizar hemostasia. Imagem pós-balonamento evidenciando sucesso no tamponamento do orifício e ausência de repercussão trombótica nos vasos distais ao local de oclusão. Realizado ultrassonografia (US) com Doppler que demonstrou hematoma sem novos sinais de expansão, sem a presença de pseudoaneurisma e sem sinais de sangramento (Figura 1).

Figura 1. Angiografia evidenciando procedimento realizado para correção de hematoma pós punção arterial no paciente do caso 1 .

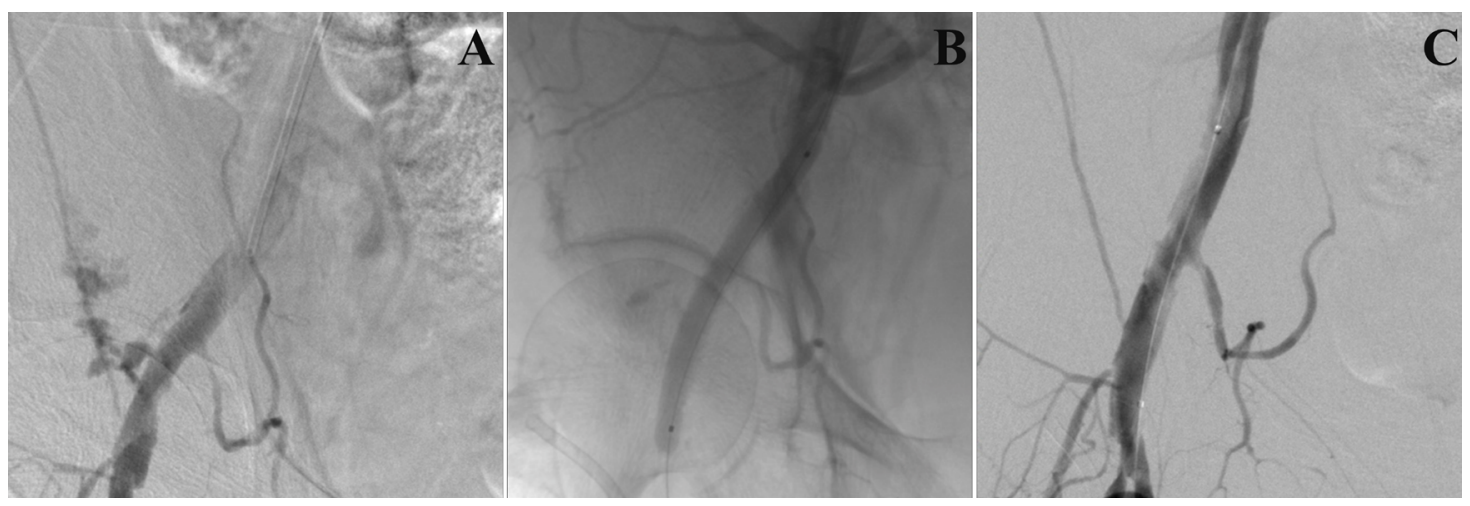

Nota: em sequência, extravasamento de contraste por orifício sangrante em artéria ilíaca externa com balonamento hemostático e imagem de controle.

\section{Caso 2}

Paciente de 63 anos, sexo feminino, hipertensa, diabética. Submetida a cateterismo cardíaco diagnóstico com punção retrógrada da artéria femoral comum direita. Evoluiu logo após a retirada do introdutor com hematoma em expansão em região inguinal direita e hipotensão, chegando a níveis pressóricos de $70 \times 40 \mathrm{~mm}$, sendo necessárias medidas de 
ressuscitação volêmica com sucesso. Após estabilização, realizou angiotomografia que evidenciou um orifício com sangramento ativo em terço proximal de artéria femoral profunda direita. Pela impossibilidade de compressão e pela indisponibilidade de stent revestido no serviço, foi optado por tentativa de hemostasia endovascular com cateter-balão de urgência em sala de hemodinâmica híbrida. Realizado punção retrógrada de artéria femoral comum contralateral com aposição de introdutor $5 \mathrm{~F}$, cateterização de artéria femoral profunda direita com guia hidrofílico 0,035 "x260 cm e cateter mamária 5F. Realizada arteriografia pelo cateter que evidenciou extravasamento de contraste por orifício de punção do cateterismo cardíaco. Posicionado cateter-balão semi-complacente $5 \times 40 \mathrm{~mm}$ em local do sangramento para tentativa de hemostasia por 3 minutos. Imagem arteriográfica de controle não demonstrou sangramento e nem oclusão arterial distal ao balonamento. US com Doppler pós procedimento evidenciou presença de hematoma sem sinais de expansão, bem como ausência de sangramento ativo e de pseudoaneurisma (Figura 2).

Figura 2. Angiografia evidenciando procedimento realizado para correção de hematoma pós punção arterial no paciente do caso 2 .

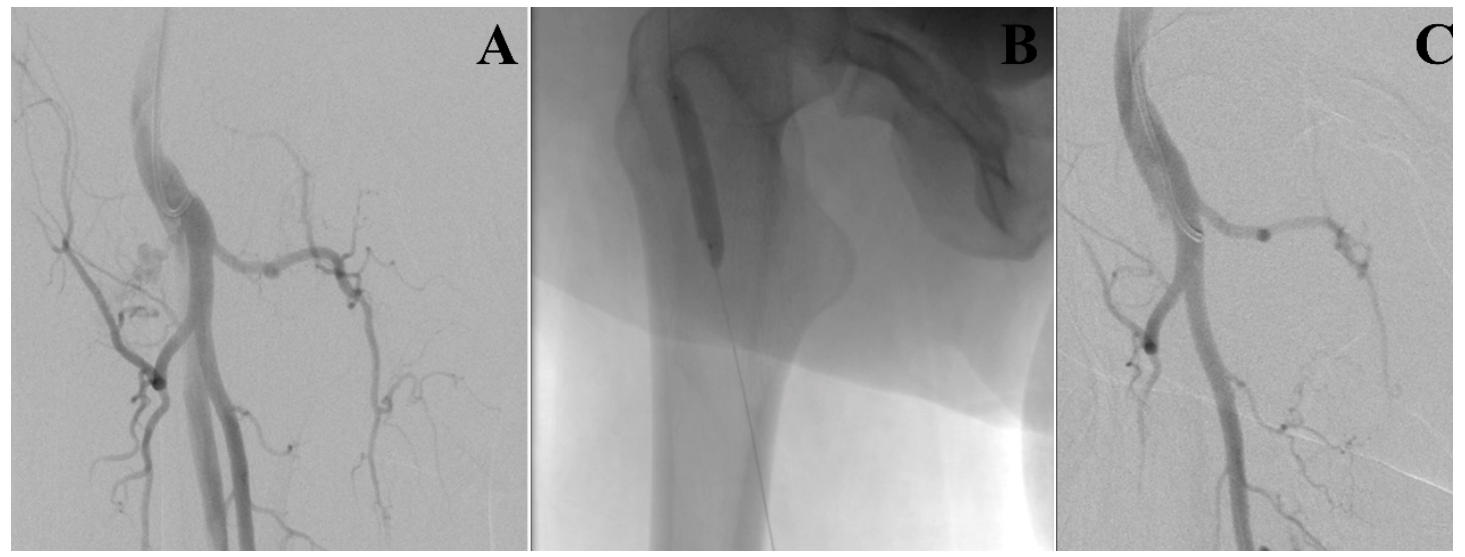

Nota: em sequência, extravasamento de contraste por orifício sangrante em artéria femoral profunda com balonamento hemostático e imagem de controle.

\section{DISCUSSÃO}

Hematomas são as complicações vasculares mais comuns dos procedimentos endovasculares. ${ }^{1,3}$ Fatores de risco apresentados pelos pacientes para estes eventos apresentam relevância e incluem: presença de hipertensão arterial, de obesidade mórbida, de disfunção renal, de aterosclerose periférica grave, punção realizada em pacientes do sexo feminino, e naqueles acima de 65 anos, bem como uso prolongado de anticoagulantes, de anti-agregantes plaquetários, de trombolíticos, e impossibilidade de manter o repouso do membro. ${ }^{1,3,5,8}$

Como fatores procedurais relacionados a sua incidência de complicações são descritos o uso de introdutores de grande calibre, tempo de procedimento prolongado, uso de doses altas de anticoagulantes, tentativas múltiplas de punção arterial, falha de dispositivos de fechamento vascular, compressão inadequada e técnica inadequada de acesso vascular, com autores relatando que até $71 \%$ destas complicações de punção na artéria femoral seriam decorrentes de punção em sítios anatômicos inapropriados. ${ }^{3,8,9}$ Ressalta-se que a punção da parede posterior do vaso causa um sangramento contínuo durante todo procedimento, sendo muitas vezes despercebido pelo realizador. No caso de uma artéria calcificada, achado frequente em pacientes portadores de DAOP como os ilustrados neste relato, sua parede é pouco elástica e pode ocorrer sangramento ao redor da bainha, principalmente quando há necessidade de anticoagulação e em procedimentos com manipulação de vários cateteres e fios guia. ${ }^{1,2,3,5,8}$

A conjunção destes fatores pode propiciar a formação de hematomas, que em sua maioria, podem ser controlados pela simples compressão manual que age tamponando o orifício sangrante e evitando sua expansão. ${ }^{1,2,3,5}$ No entanto, nos casos de grandes hematomas que causam compressões de estruturas vizinhas, contínua perda sanguínea com necessidade de transfusões, síndrome compartimental e necrose de pele, além de hematoma em expansão com o orifício sangrante não tamponado, configurando hemorragia ativa, o tratamento cirúrgico acaba sendo de escolha, sendo necessário em diversos casos a utilização de incisões convencionais, com possibilidade de ocorrência de outras complicações nãovasculares. O uso de técnicas endovasculares em casos selecionados pode ser alternativa de tratamento segura, com menores custos e taxas de morbidade, possibilitando resolução destes eventos na própria sala de hemodinâmica. . $3,3,7,9^{2}$

Nos casos apresentados, foi optado realizar uma tentativa de oclusão do orifício com cateter-balão pela impossibilidade de compressão pela anatomia dos vasos e como medida menos invasiva que a cirurgia convencional. A alternativa foi satisfatória, evitando uma conduta mais agressiva, diminuindo perdas sanguíneas com necessidade de hemoderivados, cicatrizes extensas e infecções de sítio cirúrgico. 


\section{REFERÊNCIAS}

1. Tsetis D. Endovascular treatment of complications of femoral arterial access. Cardiovasc Intervent Radiol. 2010;33(3):457-68.

2. Bhatty S, Cooke R, Shetty R, Jovin IS. Femoral vascular accesssite complications in the cardiac catheterization laboratory: diagnosis and management. Interv Cardiol. 2011;3(4):503-14.

3. Tsetis DK, Perdikakis E, Kehagias E. Percutaneous treatment of iatrogenic femoral artery access complications. Hell J Radiol. 2017;2(2):51-65.

4. Kufner S, Cassese S, Groha P, Byrne RA, Schunkert H, Kastrati A, et al. Covered stents for endovascular repair of iatrogenic injuries of iliac and femoral arteries. Cardiovasc Revasc Med. 2015;16(3):15662 .

5. Ahmad F, Turner SA, Torrie P, Gibson M. Iatrogenic femoral artery pseudoaneurysms-a review of current methods of diagnosis and treatment. Clin Radiol. 2008;63(12):1310-6.
6. Koreny M, Riedmüller E, Nikfardjam M, Siostrzonek P, Müllner M. Arterial puncture closing devices compared with standard manual compression after cardiac catheterization: systematic review and meta-analysis. JAMA. 2004;291(3):350-7.

7. Kent KC, McArdle CR, Kenned B, Baim DS, Anninos E, Skillman JJ. A prospective study of the clinical outcome of femoral pseudoaneurysma and arteriovenous fistuals induced by arterial puncture. J Vasc Surg. 1993;17(1):125-33.

8. Sherev DA, Shaw RE, Brent BN. Angiographic predictors of femoral access site complications: implication for planned percutaneous coronary intervention. Catheter Cardiovasc Interv. 2005;65(2):196-202.

9. Deser SB. Life-threatening hematoma after recurrent femoral artery puncture on a patient with massive pulmonary embolism: life-threatening femoral hematoma. International Journal of the Cardiovascular Academy. 2017;3(3-4):83-4.

\section{Como citar:}

Aguiar MA Filho, Linhares FA Filho, Andrade JE Filho, Pimentel MD, Albuquerque FJ, Leão CS Filho. Alternativa de abordagem endovascular para hematoma em expansão pós-punção arterial: relato de dois casos. Rev Med UFC. 2019 out-dez;59(4):79-82. 